\section{造ettsomian 进ectures}

\section{THE SURGICAL DISEASES OF CHILDREN.}

DELIVERED BJFORF TIIE MEDICAL SOCIETY OF J,ONDON.

IXY

T II O MAS BRYAN T, F.R.C.S., ASSISTANT-SURgEON TO GUY'S hOSPITAL.

\section{Lecture I. (Continued.)}

IIARE-Lip.

Commencing at the superior orifice of the mucous canal which forms part of the digestive system, the deformity denominated hare-lip is the most important surgical affection that demands our notice; and it is one which, without doubt, is to be explained by a distinct want or arrest of development of the fotal structure.

Anatomists are now generally satisfied that the upper jaw is developed from several centres; and that the incisive portion-the analogue of the permanent intermaxillary bone of the lower animalsexists in the human species. The development of the integument forming the lips is also modified, or is rather determined, by the development of its bony attachment ; and it is, therefore, by such an arrest of this process that a hare-lip, associated or not with a fissured alveolar process or a divided hard or soft palate, is to be explained.

A hare-lip is, therefore, a simple want of union between the tissues which are developed from different centres ; and a fissured palate, partial or perfect, is but a want of union between the osseous centre-the analogue of the intermaxillary bone of the lower animals-and the body of the superior maxillary bone.

The fissure in the soft parts or in the bone may, consequently, be either single or double, partial or complete; the extent of the deformity being determined by the period at which the arrest of the development of the parts was primarily fixed, or by the fact of the mal-development being confined to the junction of one or of both intermaxillary bones.

I might add, in passing, that if some anatomists are doubtful as to the existence of this intermaxillary bone in the human species, on account of the difficulty, not to say impossibility, of separating it from the other osseous centres in a healthy or adult bone, pathological investigations may be advanced as powerful means of proving the truth of the theory; and the following case, which is quoted from my own experience, is one in point.

A child, aged 3 years, was brought to me at Guy's Hospital in the year 1858, with necrosis of some portion of the upper jaw, which had followed upon a severe attaçik of measles. With a pair of dressing forceps the dead bone was readily removed, and subsequent convalescence followed. The bones which were removed turned out to be perfect specimens of the incisive centre of bone, or the intermaxillary; and they came away entire, fairly proving that they had been originally developed as independent cen- tres of ossification. The bones were exhibited before the Pathological Society, in the year 1858 .

We will now return to the more practical points connected with this subject of hare-lip; and I believe that I shall be able to bring before your notice some facts which cannot but be regarded as of interest; they have been adduced from the material which my opportunities at Guy's Hospital have enabled me to collect. I possess the records of sixtyfour cases of this deformity; this number including all the examples which have been admitted into Guy's Hospital for about eight years, with other cases which have fallen under my own care amongst the out-patients, these counting one-third of the whole number. A careful analysis of this material has yielded the following facts.

Nature and Extent of the Deformity. The arrest of development forming the deformity denominated hare-lip may be of different degrees of severity. It may be confined to the lip itself, or extend to the hard and soft palate, causing a mere fissure in the gum, or a complete separation of the hard and soft tissues. It may be of a single or a double nature; the double fissure, however, never extends beyond the lip and the intermaxillary bone; the cause of the deformity forming a sufficient explanation of this fact.

I have seen, in the lip of an adult, a congenital cicatrix on the left side of the upper lip, with a slight elevation of its mucous margin, which was evidently due to a condition which may be said to be the very earliest indication of a hare-lip. The child of this parent had a more complete fissure, and was operated upon by me with complete success.

'T'he different varieties and complications, however, of hare-lip will be best shown by an analysis of the collected cases; and the following facts may be relied on.

In 32 of the 64 cases, the deformity was of a simple character, and affected the lips alone to a greater or less extent ; 3 cases were complicated with a fissure of the gum, corresponding to the labial deformity; 2 had a fissured gum and hard palate ; 21 a fissured hard and soft palate; and 6 cases were of a double nature.

It is thus evident that half the cases of hare-lip are of a simple and uncomplicated character, and that in the remaining moiety some deformity of the mouth and palate coexists; a fissure of both the hard and soft palate being present in two-thirds of the remainder, or in one-third of the whole number of cases.

Seat of the Deformity. All the cases which have passed under my observation have been in the upper lip; and, although authors refer with some confidence to the fact that the lower lip may be similarly affected, it is a subject of difficulty to believe in the truth of such an opinion, as the very pathology, or rather the explanation of the nature of the deformity, forbids the idea of the possibility of such an occurrence holding any position in our minds; and if the explanation which has been just given be a true one, the opinion that it is ever found in the lower lip must be looked upon as of doubtful accuracy.

The upper lip, therefore, is its natural seat; and the next point for illustration relates to the side in which it is usually observed. That it is 
nover found in the median line, is a fact that the experience of all surgeons will at once admit; and the results of experience upon this point only bear out the truth of the explanation of its cause. That it is generally found on the left side of the body, the analysis of my own cases positively proves; for 63 per cent. of the cases were on the left side, and 36 only on the right. A satisfactory explanation of this fact has never yet been given, although I believe it to be a generally received truth that deformities, as a whole, are more common on the left than on the right side of the body; and the above figures tend in a great measure to prove the accuracy of the remark.

Sex. The influence of sex is the next point which presses upon our attention; and, if the facts which the analysis of my materials yields were not supported by the generally received impression which most surgeons entertain, they might be considered to be somewhat strange; for out of the 64 cases of hare-lip, 44 occurred in boys, and but 20 in girls, or in the proportion of 70 per cent. of the former, and 30 per cent. of the latter; this deformity occurring 40 per cent. more frequently in males than in the female sex.

If we analyse these cases again, however, an unexpected result makes its appearance; for in the simple and uncomplicated examples of harelip, the difference between the two sexes is not so great; for of the 30 cases, 18 or three-fifths were in males, and 12 or two-fifths in females. But in the more complicated examples-that is, in those in which the hard and soft palate were more or less involved - the greater frequency of its occurrence in the male sex becomes most apparent ; for of the cases in which both hard and soft palate were completely fissured, 17 cases, or 80 per cent., were in boys, and but 4 cases, or 20 per cent., in girls; the male elemest predominating to an extreme degree. $\therefore$ nd in "ite worst cases of all-that is, of double havi-lip..-all the six examples were observed in male chicicos.

These facts are, to say the least, extremely curious ; for it can hardly, I think, be looked upon as an accident that, in the cases which have passed under my own observation, the male element should have predominated so largely; and I am disposed to look upon my own experience as an expression of what would be that of others, if it were given to us. By way of summary, the following conclusions may be drawn.

Conclusions. 1. Hare-lip is always found in the upper lip, and is most frequent on the left side of the body ; 63 per cent. of the cases taking place on the left side, and 36 per cent. on the right.

2. It is more common in the male than in the female sex, in the proportion of 70 per cent. of the former to 30 per cent. of the latter.

3 . In the simple uncomplicated cases, the proportion is less marked; but as the cases become more complicated, the greater frequency of its occurrence in the male sex becomes more apparent; the proportion between the sexes being 80 per cent. and 20 per cent. It would also appear to be a rare thing to find a double hare-lip in a female child.

Treatment of Hare-Lip. There neither is nor can be any difference of opinion between surgeons as to the expediency of affording surgical relief in the cases of deformity which we are now considering; although there is still great uncertainty and diver- sity of opinion between them as to the period at which it is the most advisable to submit the child to an operation. Some surgeons assert that operative interference is most successfully carried out at the very earliest period of the child's existence; and that the earlier the operation is performed, the greater are the chances of a complete success. On the other hand, surgeons of equal eminence maintain that the practice just laid down is fraught with danger, and that the correct practice is to wait till the child's powers are fairly established; and that one or even two years should be allowed to pass before any attempt to repair the deficiency should be made. Between these two extremes lie the opinions of many men; and it is, therefore, with a view of obtaining some definite data upon which a positive opinion can be based, that I will now lay before you the conclusion to which I have been led.

But, before doing so, allow me to allude with all deference to the uncertain grounds upon which surgeons have been in the habit of founding positive opinions; for it is too true that these opinions are, as a rule, based only upon impressions; although it may be that these impressions arc the result of extensive and perhaps most conscientious experience. Still I feel that I may appeal with confidence to all who hear me, when I assert that there are few things more fallacious than the general impressions which experience affords, however great may have been the field from which they have been gleaned; and that true impressions can only be gained from positive and definite materials.

I am unable to direct you to any work or even journal in which definite data have been recorded, from which just conclusions can be drawn as to the period at which it is the most advisable to operate in cases of hare-lip, unless I were to mention my own previous contributions to this subject, which will be found in the third part of my Clinical Surgery.

It is, therefore, with some confidence that I am now able to add something to our stock of knowledge which may prove of use in guiding us to a correct decision. If I lay myself open to the charge of saying that surgeons have hitherto formed their opinions upon indistinct and indefinite data, I can only justify myself by pleading that the grounds upon which the opinions have been based have not been published; and that, therefore, I may be forgiven for doubting their existence.

The facts which I now adduce may form but a nucleus round which others may hereafter collect; and the conclusions I now give may have to be confirmed or corrected by future observers.

Analysis of Cases. Four cases were operated on within the first two weeks; one on the fifth day, and two on the eleventh day, one of which proved fatal.

Eight cases were between four and five weeks of age ; in two of these, the parts subsequently gave way, although the cases in the end turned out well.

'Ten cases were operated on between the sixth and eleven weeks, with success.

Fifteen cases were treated between the third and sixth month, with only one failure.

Six cases were operated on between the sixth and twelfth month, with a good result; and

Fifteen were also successfully treated after the first year.

From these facts it will appear that, during the first six weeks of life, the operation for hare-lip is 
by no means so successful as to warrant its general performance, unless an absolute necessity compels. Out of the twelve cases, one died; and in two the wound subsequently sloughed, although in both a good recovery was finally secured. At a later period of life, a successful issue was recorded in all.

At what period of the infant's life, then, should the operation for hare lip be generally performed? In order that a satisfactory answer should be given to this question, it is necessary to consider the purposes for which the operation may be required; and they may be divided into two classes.

Firstly, the operation may be called for to preserve life; the imperfection of the mouth forming an insuperable difficulty to the child's sucking.

Secondly, it may be called for simply as an operation of expediency, to improve appearances and remove defects.

When demanded for the satisfaction of the first purpose-namely, to preserve life-there is no question of period which the surgeon has to decide. The opcration must be undertaken at all hazards; but the successful termination of the case will be uncertain. For this purpose, I have performed the operation on the fifth day with complete success, and other surgeons have been equally successful even at a much earlier period; but, under these conditions, the necessity of the operation overcomes all other questions, and the surgeon has no option but to do his best.

In the second and more numerous class of cases, however, the time for operation rests entirely on the surgeon's will ; and it only remains for us to decide from the records of positive experience, the period of life at which the greatest success can be anticipated.

Judging from the materials which I have but just laid before you, it would appear that the operation when performed at any period of life after the sixth week is likely to be followed by a successful result; I would give the preference, however, to about the third month of life, the vital powers of the child having by that time become fairly established, and well able to resist the tax upon their strength which is necessarily occasioned by any operation. At an earlier date-that is, during the first few weeks of life-the operation should be condemned; unless, as it has been previously explained, the existence of the child appear imperilled.

The Operation. It will be hardly profitable to any of us if I were to take up your time by recapitulating all the varieties of operation which have been suggested by various surgeons for the relief of the deformity now under consideration; and I propose, therefore, to confine my remarks simply to what I have observed and practised, and to recommend what appears to be the best means out of the many which have received attention.

In simple cases, there are but two main oljects which the surgeon has in view-to pare the edges of the fissure, and to adapt them so as to render the deformity as slight as possible.

I would give the preference to the scalpel for the performance of the first stage ; for with such an instrument a cleaner section of the tissues is made than when scissors are employed; and this cleanness of the incision is a point of primary importance.

The form of section which is to be recommended is variously estimated by different surgeons. Some are content with a clean straight section of the lip's margin, being so satisfiad with obtaining simple union of the separated parts, as not to care about making an attempt to supply the deficiency of tissue which is invariably present at the margin of the lips. Other surgeons recommend that the line of incision should be curved inwards, so that the lower margin of the lips, when brought into position, will be made to project downwards, and thus tend to correct the evil which has been just alluded to. But I am disposed to give the preference to the plan of operation which I believe was originally suggested by Malgaigne, as by it the labial notch is almost to a certainty done away with, and the deformity most completely remedied. It consists simply in paring the edges of the wound from above downwards, leaving the inverted flap adherent at its labial border. When this is done, and the upper edges of the divided lips have been brought together, the lower flaps may be connected by a fine suture; and, if proved to be too long, they may be curtailed; sufficient material being left to fill in the gap which is too often the result of the other forms of operation. In my hands, this plan of operation has proved eminently successful, and it only requires in its application a little nicety in the adjustment of the parts.

An important preliminary point, however, demands attention, as the success of the case rests materially upon its due performance; for, however well the marginal incisions may be cxecuted, unless the lip have been rendered readily moveable upon its labial attachments, a failure to the operation must be expected. This freedom of movement is, howcver, readily secured by making a free section of the mucous membrane of the lip from its osseous connexions. When this is done, the whole lip can be readily raised from its position, and all chances of tension are completely taken away.

'The fear of removing too much of the lip's margin is ore which is most certainly groundless, the usual fault of operating surgeons lying the other way ; for the labial tissues are very extensible, and a free section of the parts is to be preferred.

The second step of the operation remains for us to describe ; its object being to bring the edges of the wound together, and to keep them there. This end is most readily and efficiently carried out by means of the interrupted suture. Silk or wire may be selected according to the fancy of the operator, one exciting as much or as little irritation as the other. 'The interrupted suture is to be preferred to the uninterrupted, as it is more readily removed.

The sutures should be inserted from a quarter to half an inch from the wound's margin, and carried obliquely through the lip, to the line which is bounded by the mucous membrane, but not through it, and then introduced at a like spot on the opposite side, and firmly tied. A fine suture at the labial margin should also be applied, in order to maintain as accurately as possible the line of lip.

All bleeding is generally at once arrested when the wound's margins are brought into contact; but, if it be troublesome, one of the sutures may be made to perforate the bleeding vessel.

A little elastic collodion may be subsequently painted over the part, to prevent friction; but, as a rule, the practice of leaving the part open proves equally satisfactory. The employment of pins in 
simple cases may certainly be looked upon as unrecessary, as the simple means which have been described are amply sufficient.

In operating, therefore, upon uncomplicated cases of hare-lip, the following points appear the most important.

First, to separate the lip freely from its gummy attachments; secondly, to make a free section of its edges, according to the plan previously laid down; and thirdly, to bring the edges accurately together by fine interrupted sutures, introduced at a distance from the wound's margin, and deeply placed. If these points are observed, and the child is neither too young nor too sickly, a successful termination to the case may with some confidence be predicted.

Complicated Cases of Have-lip. The remarks which I have just concluded, concerning simple and uncomplicated hare-lip, are equally applicable to other cases of a more complicated nature; but there is a point of practice in double hare-lip which deserves a few moments' attention, as there is still a diversity of practice in such cases which, I confess, appears to me somewhat singular. Some surgeons invariably operate only upon one side at a time, fancying that a greater success follows such a line of practice than when both sides are treated at one operation. From what has passed under my observation, I cannot see any reason why both sides should not be simultaneously treated; but, on the other hand, believe that advantages are obtained by such a proceeding, which are not secured by the more timid and prolonged process. In the six examples of double hare-lip which I have tabulated, primary success followed in each instance; and in the cases which fell into my own charge, I had no reason to believe that the operation would have succeeded better if a different practice had been followed. In one case of a boy aged nine weeks, I employed a pin in order that the centre bit should be well held down to the lateral portions; and good success followed the attempt. In the second case, of a boy aged one month, I operated after the plan which I have previously given; but I preserved only the lateral flaps of the outer portions, joining these in the centre beneath the central piece. Primary union followed the operation, and a recovery in which the deformity was but little marked.

In no instance have I witnessed any evils resulting from the practice which I have just laid down; and I am at a loss to understand the principle upon which surgeons still adhere to the older practice of carrying out a double and separate operation.

In certain complicated cases of hare-lip, the central incisive or intermaxillary portion of bone is found projecting to a detrimental extent; and it becomes a question with the surgeon whether he shall remove it by means of bone-forceps, or fracture it and press it backwards. In many instances, unless some such proceedings as these be carried out, the operation for hare-lip cannot be performed. I have seen both plans executed with good success in the practice of my colleagues, and in one instance have performed excision with success.

If the bone can be pressed backwards by a moderate amount of force, the practice appears to be preferable to the one in which the obstacle is only overcome by its removal; but, if this plan should be impossible or inexpedient, there is no alternative but to excise the part. It is true that, hy removing the central portion, the incisor teeth will be taken away ; but it is better that such a practice should be carried out, than that the original deformity should be left, particularly when it is remembered that, with such a projecting bone, the incisor teeth would in all probability appear much out of place, even to the extent of piercing the lip-a contingency which I have in one case certainly observed.

In some instances, however, an exfoliation of the incisor tooth may take place before any operative interference has been performed; and this was illustrated in a case which fell under my care on Oct. 27 th, 1862, in a male child aged one month, who had a hare-lip on the left side, with a fissured hard and soft palate, the incisive portion of the superior maxillary bone projecting far forwards. When the mother brought the child to me, the soft parts over the projecting bone were dry and apparently dead ; and with the slightest touch the "scab" came off, and with it the crown of the incisor tooth. The surface readily healed; and the child is to return to me for operation at a future period.

There is one other point in the treatment of these cases which the surgeon should rernember; and that is, if the primary operation fail, and primary union cannot be procured, there is still a good hope of securing ultimate success by union from secondary adhesion. This end is to be obtained by the application of strapping, or in preference sutures, to the granulating wound, so that they may again be placed in apposition. In several instances which have been tabulated, this practice was performed, and a good result took place; and in two cases which came into my hands several years ago, equal success may be recorded. Both were infants, upon whom I had been led to operate at a very early period, two and three weeks only being their respective ages. Sloughing followed the original operation; and when the parts had begun to granulate, sutures were reapplied, and a good recovery was eventually secured.

With these remarks I will now conclude the subject of hare-lip, having, I believe, touched upon the chief points of practical importance, and given facts upon which definite opinions may be based. The personal experience of any single surgeon in hare-lip is rarely very great; and it can only be by the collective experience of many that positive opinions can be based. I advance my own facts, knowing them to be true; and entertain the hope that other surgeons may be induced to add their own stock of cases to these which I now contribute, and thus either confirm or correct the opinions which have been based upon them.

The subject of cleft palate is not one which need occupy our attention; for, although it is a congenital defect, it can be remedied only in advanced life, and has, therefore, no special claim for notice. Artesia oris or closure of the mouth is also a congenital condition which may exist; but, as I have had no personal experience upon the subject, it will receive only this passing notice.

\section{[To be continued.]}

The Edinbligh Childies's Hospital. The Queen has been graciously pleased to become patroness of this charity, and has intimated, through the Secretary of State, her desire that it be henceforth called "T The Royal Fdinburgh Hospital for Sick Children'. 